\title{
Calpain-Regulated p35/cdk5 Plays a Central Role in Dopaminergic Neuron Death through Modulation of the Transcription Factor Myocyte Enhancer Factor 2
}

\author{
Patrice D. Smith, ${ }^{1}$ Matthew P. Mount, ${ }^{1}$ Raj Shree, ${ }^{1}$ Steve Callaghan, ${ }^{1}$ Ruth S. Slack, ${ }^{1}$ Hymie Anisman, ${ }^{2}$ Inez Vincent, ${ }^{3}$ \\ Xuemin Wang, ${ }^{4}$ Zixu Mao, ${ }^{4,5}$ and David S. Park ${ }^{1}$ \\ ${ }^{1}$ Ottawa Health Research Institute, Ottawa, Ontario, Canada K1H 8M5, 2Neuroscience Institute, Carleton University, Ottawa, Ontario, Canada K1S 5B6, \\ ${ }^{3}$ Department of Pathology, University of Washington, Seattle, Washington 98195 , and Departments of ${ }^{4}$ Pharmacology and ${ }^{5}$ Neurology, Emory University \\ School of Medicine, Atlanta, Georgia 30322
}

The mechanisms underlying dopamine neuron loss in Parkinson's disease (PD) are not clearly defined. Here, we delineate a pathway by which dopaminergic loss induced by 1-methyl-4-phenyl 1,2,3,6 tetrahydropyridine (MPTP) is controlled in vivo. We reported previously that calpains play a central required role in dopamine loss after MPTP treatment. Here, we provide evidence that the downstream effector pathway of calpains is through cyclin-dependent kinase 5 (cdk5)-mediated modulation of the transcription factor myocyte enhancer factor 2 (MEF2). We show that MPTP-induced conversion of the cdk5 activator p35 to a pathogenic p25 form is dependent on calpain activity in vivo. In addition, p35 deficiency attenuates MPTP-induced dopamine neuron loss and behavioral outcome. Moreover, MEF2 is phosphorylated on Ser444, an inactivating site, after MPTP treatment. This phosphorylation is dependent on both calpain and p35 activity, consistent with the model that calpain-mediated activation of cdk5 results in phosphorylation of MEF2 in vivo. Finally, we provide evidence that MEF2 is critical for dopaminergic loss because "cdk5 phosphorylation site mutant" of MEF2D provides neuroprotection in an MPTP mouse model of PD. Together, these data indicate that calpain-p35-p25/cdk5-mediated inactivation of MEF2 plays a critical role in dopaminergic loss in vivo.

Key words: cyclin-dependent kinase 5; cdk5; myocyte enhancer factor 2; MEF2; dopamine; substantia nigra; Parkinson's disease; 1-methyl-4-phenyl-1,2,3,6-tetrahydropyridine; MPTP

\section{Introduction}

Parkinson's disease (PD) is a progressive neurological condition characterized by loss of dopamine neurons in the substantia nigra pars compacta (SNc) (Dauer and Przedborski, 2003). The 1-methyl-4-phenyl-1,2,3,6 tetrahydropyridine (MPTP) model recapitulates many of the pathological features of PD (Dauer and Przedborski, 2003) and is commonly used to decipher signaling mechanisms underlying dopaminergic degeneration in vivo. Our previous work with this model indicates that the cysteine protease calpain is central to dopamine neuron loss (Crocker et al., 2003). The relevance of this data to PD pathogenesis is supported by the observation that calpain expression and activity is elevated in postmortem PD brains (Mouatt-Prigent et al., 1996; Crocker et al., 2003).

Calpains are calcium-dependent cysteine proteases that have

Received July 13, 2005; revised Nov. 7, 2005; accepted Nov. 8, 2005.

This work was supported by grants from the Canadian Institutes of Health Research (CIHR), Parkinson's Society Canada, Parkinson's Disease Foundation, Michael J. Fox Foundation, and Parkinson's Research Consortium. H.A. is a Canada Research Chair in Neuroscience. P.D.S. is a CIHR doctoral research award recipient. Z.M. was supported by National Institutes of Health Grants HD39446 and NS048254.

Correspondence should be addressed to Dr. David S. Park, Ottawa Health Research Institute-Neuroscience, University of Ottawa, 451 Smyth Road, Ottawa, Ontario, Canada K1H 8M5. E-mail: dpark@uottawa.ca. D0I:10.1523/JNEUROSCI.2875-05.2006

Copyright $\odot 2006$ Society for Neuroscience $\quad 0270-6474 / 06 / 260440-08 \$ 15.00 / 0$ been implicated in several cellular functions (Melloni et al., 1992; Carafoli and Molinari, 1998; Goll et al., 2003; Ray and Banik, 2003). Calpain activity is regulated by calpastatin, a ubiquitously expressed endogenous inhibitor (Melloni et al., 1992; Croall and McGrody, 1994). A growing list of possible physiological substrates of calpains have been described previously (Carafoli and Molinari, 1998; Chan and Mattson, 1999; Goll et al., 2003). However, substrates relevant to dopaminergic neurodegeneration are unknown. In this regard, we performed experiments to identify critical downstream targets of calpain activation and define mechanistically how these targets are regulated in dopamine neurons after MPTP treatment. Of particular interest is the observation that calpains can potentially mediate the proteolysis of the cyclin-dependent kinase 5 (cdk5) activator p35 (Kusakawa et al., 2000; Lee et al., 2000). This observation is made more significant by our findings that cdk5 mediates dopamine neurodegeneration in vivo (Smith et al., 2003).

Cdk5 is a member of the family of cyclin-dependent kinases (CDKs) (Pines, 1993). In contrast to the classical cell cyclerelated CDKs, cdk5 is predominantly involved in regulation of postmitotic neuronal signals (Tsai et al., 1993, 1994) and requires association with its regulatory partners, p35 (Tsai et al., 1994) or p39 (Ko et al., 2001), to be active. Cdk5 has been implicated in regulation of several neuronal processes including neurodevel- 
opment (Ohshima et al., 1996; Ko et al., 2001), axonal transport (Julien and Mushynski, 1998), synaptic activity (Rosales et al., 2000), and dopamine signaling (Nishi et al., 2002; Chergui et al., 2004). Although cdk5 is involved in regulation of normal physiological function, correlative evidence now suggests that this protein may be usurped to signal death (for review, see Shelton and Johnson, 2004; Smith et al., 2004). However, the mechanism by which $\mathrm{cdk} 5$ regulates neurodegeneration is not clearly defined. Importantly, conclusive molecular evidence of the functional importance of $\mathrm{p} 35 / \mathrm{cdk} 5$ in any adult injury setting in vivo is lacking. Although several groups have reported that cdk5-related signaling is altered in various animal models of neuronal death (Patrick et al., 1999; Nguyen et al., 2001; Bu et al., 2002), the significance of this was unclear. To support the required death-promoting role of cdk5 in vivo, we recently reported that dominant negative (DN) cdk5 expression blocks dopaminergic loss after MPTP treatment (Smith et al., 2003). Although this is suggestive of the importance of abnormal cdk5 activity, it is possible that DN cdk5 expression might also block non-cdk5-specific pathways.

In this study, we provide more conclusive molecular evidence of the importance of cdk5 in MPTP-induced dopaminergic death and show that the cdk5 regulator p35 is central to MPTP-induced loss. In addition, we provide pathways that act both upstream and downstream of cdk5. MPTP induces calpain-dependent formation of p25, a cleavage product of p35, resulting in increased cdk5 activity in the substantia nigra. Importantly, p35/p25 deficiency attenuated dopaminergic loss, improved markers of basal ganglion function, and improved MPTP-induced behavioral deficits. We also found that calpain-mediated regulation of p35/cdk5 increased phosphorylation/inactivation of the survival factor myocyte enhancer factor 2D (MEF2D). Cdk5-mediated phosphorylation of MEF2D, on the inactivating Serine 444 residue, played an integral role in dopamine neuron loss, because adenoviral mediated expression of MEF2D (S444A) protected dopamine neurons from MPTP-induced insult. These data provide the first evidence to support a mechanistic role for a calpain-p35-25/cdk5-MEF2 pathway in dopamine neuron loss associated with PD.

\section{Materials and Methods}

Experimental animals. Male C57BL/6 mice (22-28 g body weight; Charles River Laboratories, St. Constant, Quebec, Canada) were used for all experiments (unless otherwise stated). p35-deficient mice (bred on a C57BL/6 background) and wild-type littermates were generated by breeding heterozygote p35 mutants. Genotyping information was reported previously (Hallows et al., 2003). All animal experiments conformed to the regulations set out by the Canadian Institutes of Health Research and the Canadian Council for Animal Care, with approval from the University of Ottawa Animal Care Committee. MPTP ( $25 \mathrm{mg} / \mathrm{kg}$, i.p.; measured as free base; MPTP-HCl; Sigma, St. Louis, MO) was administered to mice once a day for five consecutive days as first described by Tatton and Kish (1997). This model has also been reported previously (Crocker et al., 2003; Smith et al., 2003).

Delivery of adenoviruses. Adenoviral constructs were delivered unilaterally to the right striatum using coordinates as described previously (Crocker et al., 2003). Briefly, each adenovirus [Ad.CalSt, adenovirus overexpressing calpastatin; Ad.MEF2D (S444A), cdk5 phosphorylation mutant of MEF2D; and Ad.MEF2CVP16, constitutively active form of $\mathrm{MEF} 2 \mathrm{C}]$ was injected into the right striatum $7 \mathrm{~d}$ before initiation of MPTP or saline treatment. Either a LacZ-containing construct or a GFPcontaining construct was used as controls for all adenovirus experiments. A single unilateral injection of virus was given to each animal $(2 \mu \mathrm{l} ; 1 \times$ $10^{7}$ particles $\left./ \mu \mathrm{l}\right)$, delivered to the right striatum $(0.5 \mathrm{~mm}$ rostral, $2.2 \mathrm{~mm}$ right of bregma, and $3.4 \mathrm{~mm}$ below the skull surface). Each adenovirus injection was given at a constant rate of $0.5 \mu \mathrm{l} / \mathrm{min}$ using a syringe pump system (Harvard Apparatus, Holliston, MA). Expression of adenoviral protein in the nigral region was validated $7 \mathrm{~d}$ after administration of the virus by both Western blot and immunohistochemistry. Seven days after viral injection, animals were treated with MPTP as described above, and brains were extracted at indicated times as described below.

Immunohistochemistry/immunofluorescence. For immunohistochemical analysis, mice were transcardially perfused with $0.9 \%$ saline followed by an equal volume of buffered $4 \%$ paraformaldehyde (PFA). Brains were removed from the skull, postfixed in $4 \%$ PFA, and then cryoprotected in sucrose solution before cryosectioning. Brains were sectioned in the coronal plane at $14 \mu \mathrm{m}$ thickness and collected either free-floating or on slides. Immunohistochemical analysis was performed as described previously (Crocker et al., 2003; Smith et al., 2003). Briefly, coronal sections were incubated overnight at $4^{\circ} \mathrm{C}$ in specific primary antibody [monoclonal tyrosine hydroxylase (TH), 1:1000 (ImmunoStar, Hudson, WI); rat polyclonal dopamine transporter (DAT), 1:500; rat polyclonal MEF2D, 1:1000 (Santa Cruz Biotechnology, Santa Cruz, CA); rabbit polyclonal p35 (C-19) (Santa Cruz Biotechnology); pMEF2D (S444), 1:1000 (Gong et al., 2003); or MEF2, 1:1000 (Santa Cruz Biotechnology)]. Following primary antibody, sections were washed in PBS and then incubated in an appropriate biotinylated secondary antibody (1: 200; Jackson ImmunoResearch, West Grove, PA) overnight at $4^{\circ} \mathrm{C}$. Sections were then washed in PBS and incubated in a streptavidin horseradish peroxidase (HRP) tertiary antibody (1:200; Amersham Biosciences, Arlington Heights, IL). Immunohistochemical staining was visualized using a diaminobenzidine/glucose oxidase reaction (Crocker et al., 2003; Smith et al., 2003). Photomicrographs were taken using the Northern Eclipse software with an Axiophot camera connected to a Zeiss (Thornwood, NY) microscope system.

Western blot analysis. Western blot analysis was performed on protein samples extracted from nigral tissue. Nigral tissue samples were extracted from a $2 \mathrm{~mm}$ slab of tissue using a biopsy needle. Protein was extracted as described previously (Smith et al., 2003). Protein samples were resolved on SDS-PAGE gel and transferred to nitrocellulose membrane. Membrane was incubated in primary antibody [monoclonal TH, 1:500 (ImmunoStar); rat polyclonal MEF2D, 1:500 (Santa Cruz Biotechnology); rabbit polyclonal p35 (C-19), 1:1000 (Santa Cruz Biotechnology)]. Primary antibody incubation was followed by washing in a $1 \times \mathrm{PBS} / 0.1 \%$ Tween 20 solution and then incubating in a secondary HRP antibody (1:2000; Bio-Rad, Hercules, CA). A chemiluminescence reaction (Amersham Biosciences) was used to visualize protein bands on Kodak (Rochester, NY) film.

Cdk5 kinase assay. Cdk5 kinase assay was performed on nigral tissue samples as described previously (Smith et al., 2003). Briefly, protein was extracted from samples dissected from the substantia nigra region. Protein samples $(50 \mu \mathrm{g})$ from the SNc of saline and MPTP-treated animals were incubated overnight in $\mathrm{Cdk} 5(\mathrm{C}-8,1 \mu \mathrm{g}$ of antibody per sample; Santa Cruz Biotechnology). Thirty microliters of $50 \%$ slurry of Protein A-Sepharose (Sigma) were then added to immunoprecipitates, and samples were incubated for $2 \mathrm{~h}$ at $4^{\circ} \mathrm{C}$. The immune complexes were then pelleted at $14,000 \times g$, washed in kinase buffer containing $20 \mathrm{mM} \mathrm{HEPES}$, $\mathrm{pH}$ 7.6, $20 \mathrm{~mm} \mathrm{MgCl}_{2}, 20 \mathrm{~mm} \beta$-glycerophosphate, $0.1 \mathrm{~mm} \mathrm{Na}$ orthovanadate, $2 \mathrm{~mm}$ DTT, and $20 \mu \mathrm{M}$ unlabeled ATP. Each sample was then incubated in the above-described kinase buffer containing $2 \mu \mathrm{g}$ of Histone $\mathrm{H} 1$ and $1.2 \mu \mathrm{Ci}\left[\gamma^{-32} \mathrm{P}\right] \mathrm{ATP}$ at $30^{\circ} \mathrm{C}$ for $20 \mathrm{~min}$. Kinase activity was determined by SDS-PAGE and autoradiography.

HPLC. HPLC analysis was used to evaluate the levels of dopamine and dopamine metabolites in nigral and striatal samples extracted from both saline-treated and MPTP-treated animals. HPLC analysis was performed as described previously (Hayley et al., 2001). $\mathrm{MPP}^{+}$measurement was performed as described previously (Hayley et al., 2004).

Dopamine neuron survival. Dopamine neuron survival was analyzed by immunohistochemical assessment of TH-positive cells in the substantia nigra pars compacta, (anatomical regions were defined as per Paxinos mouse brain atlas) (Franklin and Paxinos, 2001). A positive counted neuron was one that was located in the $\mathrm{SNc}$ region, $\mathrm{TH}$ positive, process bearing, and nucleated. In addition to evaluating TH-positive cells, an independent histological marker, cresyl violet (CV), was used to validate TH cell counts as described previously (Crocker et al., 2003). For knock- 
out animals, total number of neurons was estimated using Abercrombie's correction method (Abercrombie, 1946) on serial sections as described previously (Crocker et al., 2003; Smith et al., 2003). For this correction, the size of counted neuronal structure was determined by averaging the diameter of the nucleated TH-positive cell soma of randomly selected neurons of the unlesioned nigra. Cresyl violet staining and cell counts were performed at the level of the medial terminal nucleus (MTN). For adenoviral gene delivery experiments, only sections containing the MTN were counted. This region has been shown previously to contain the highest level of viral protein expression after adenoviral delivery at the described coordinates (Crocker et al., 2001). At least three sections per anatomical level were analyzed per animal. The data are represented as ipsilateral (treated side) for all treatment groups; however, both treated and untreated sides were analyzed. The untreated side was used as an internal control.

Striatal densitometry. Quantification of DAT staining was performed on striatal tissues from animals $14 \mathrm{~d}$ after MPTP treatment. Counts were made by sampling an area of $660 \times 800 \mu \mathrm{m}$ in at least three sections per animal using computer-assisted image analysis software (Northern Eclipse; Empix Imaging, Mississauga, Ontario, Canada) as described previously (Crocker et al., 2003). Background cortical staining was subtracted for each section and used as an internal control.

Behavioral analysis. Behavioral analysis was performed by using the computer-assisted beam break, MicroMax system (Accuscan, Columbus, $\mathrm{OH}$ ). Animals were monitored for a $24 \mathrm{~h}$ period before initiation of MPTP treatment and $7 \mathrm{~d}$ (monitoring started on day 6) and $14 \mathrm{~d}$ (monitoring started on day 13) after the first MPTP injection as described previously (Hayley et al., 2004).

Statistical analysis. All statistical analysis was performed by using oneway ANOVA followed by Newman-Keuls post hoc test (unless otherwise stated).

\section{Results \\ Calpain-mediated generation of p25 is associated with increased cdk5 activity in the substantia nigra after MPTP treatment}

To determine the effect of MPTP-induced calpain activation on modulation of the p35/cdk5 complex, we first examined the expression of $\mathrm{p} 35 / 25$ in the substantia nigra region by Western blot analysis using a C-terminal polyclonal antibody that recognizes full-length p35 as well as the cleaved product, p25. As shown in Figure 1, MPTP treatment induces generation of p25 in the substantia nigra region (Fig. $1 \mathrm{~A}$ ), similar to what we have reported previously (Smith et al., 2003). p35 levels did not appear to be significantly changed, however (data not shown), suggesting that only a small percentage of p35 contained in a gross nigral tissue extract is converted after MPTP treatment. To examine the effect of calpain inhibition on p25 expression, we used an adenoviralmediated approach to express the endogenous calpain inhibitor, calpastatin (Ad. CalSt), that selectively inhibits calpains in the substantia nigra. We previously reported that this adenoviral calpastatin can selectively target the substantia nigra, was expressed in dopaminergic cells after striatal adenovirus injection, and protected dopaminergic neurons from MPTP-induced insult (Crocker et al., 2003). Western blot analysis revealed that p25 generation in the substantia nigra region was significantly reduced in the presence of calpain inhibition (Fig. $1 A$ ). Double immunofluorescence analysis with monoclonal TH and p35 (Cterminal) antibodies revealed that p35/25 is expressed in dopaminergic cell bodies (data not shown). However, because there is no antibody presently available to distinguish between p35 and p25 by immunofluorescence, we could not confirm by colabeling whether the increase in p25 occurred exclusively in dopamine neurons. Quantitative densitometric analysis of Western blots $(n=3)$ for $\mathrm{p} 25$ expression revealed a persistent MPTP-induced formation of p 25 in the nigral region, which was reduced in the
A
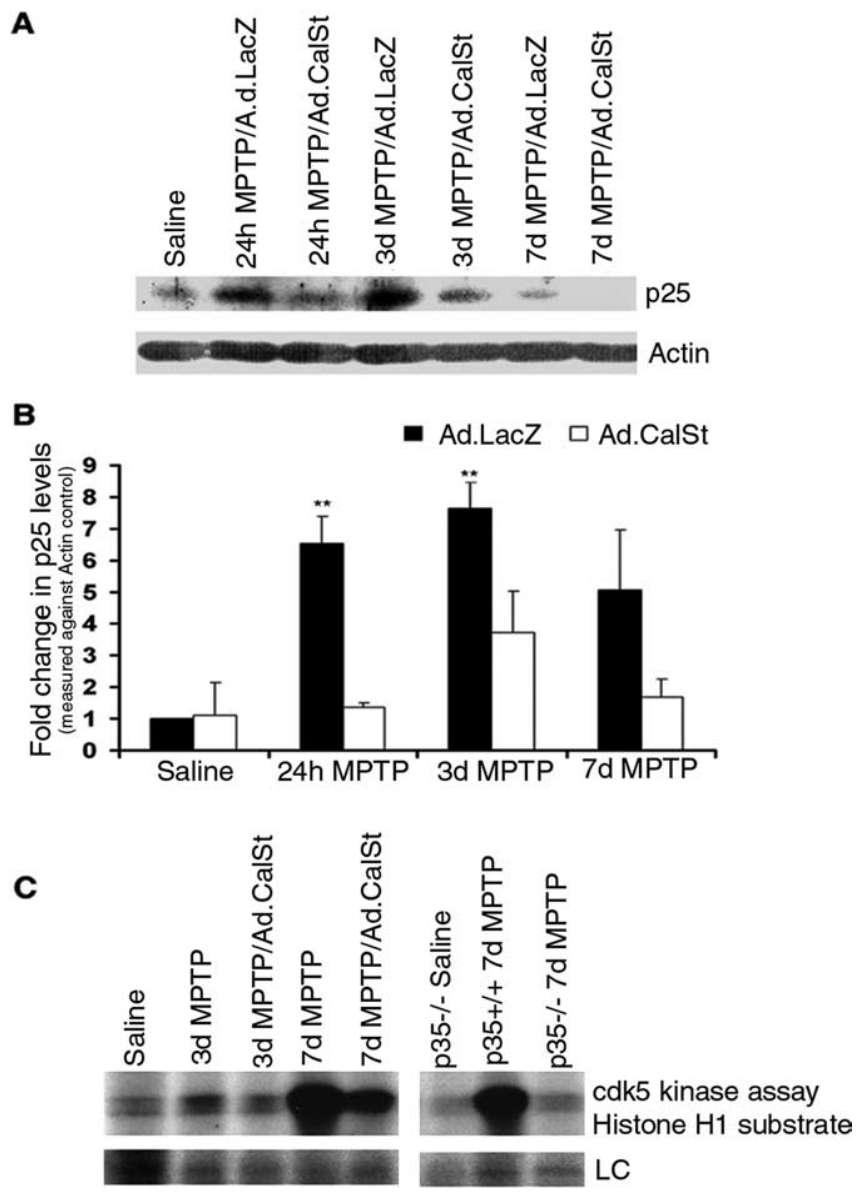

Figure 1. MPTP induces calpain-dependent formation of p25 and increase in cdk5 activity. $\boldsymbol{A}$, Representative Western blot showing MPTP-induced increase in p25 expression. Protein samples were extracted from the nigral region of mice treated with either calpastatin adenovirus (Ad. CalSt) or LacZ control. Calpastatin-treated animals showed significant reduction in MPTP-induced p25 levels compared with LacZ controls. $\beta$-Actin was used as a protein loading control. B, Quantification of $\mathrm{p} 25$ band intensity ( $\sim 25 \mathrm{kDa}$ molecular weight) measured against $\beta$-actin protein loading control at $24 \mathrm{~h}, 3 \mathrm{~d}$, and $7 \mathrm{~d}$ after MPTP treatment ( $n=3$ per treatment group) with calpain inhibition (Ad. CalSt) or LacZ control (Ad. LacZ). C, Representative kinase assay of nigral protein extracts subject to in vitro cdk5 kinase assay, using Histone $\mathrm{H} 1$ as the substrate. Cdk5 activity is significantly increased $7 \mathrm{~d}$ after MPTP treatment. MPTP-induced increase in cdk5 activity is attenuated by calpain inhibition (Ad. CalSt.) and p35 deficiency. Loading control (LC) is Coumassie blue-stained band in destained kinase assay gel. Similar results were obtained in two independent experiments. Error bars represent \pm SEM.

presence of adenoviral calpastatin expression after MPTP treatment (Fig. $1 B$ ). Because p35 is a critical activator of $\mathrm{cdk} 5$, and given the calpain-dependent modulation of this signal after MPTP treatment, we evaluated the consequence of calpaindependent p35 cleavage on cdk5 kinase activity. As shown in Figure 1, MPTP induced a robust increase in cdk5 activity in the nigral region at $7 \mathrm{~d}$ after MPTP treatment. This increase in cdk5 activity was partially attenuated by adenoviral-mediated calpain inhibition (Fig. 1C). The importance of a p35/25-mediated response in this process was substantiated by the observation that nigral extracts from $\mathrm{p} 35$-deficient mice also displayed significant reduction in MPTP-induced cdk5 activation (Fig. $1 C$ ). Interestingly, the time course of p 25 formation does not completely overlap with that of cdk5 activation. This suggests that other events, such as phosphorylation modifications (Sharma et al., 1999; Hallows et al., 2003; Rosales et al., 2003), are likely also important for cdk5 activity. However, because cdk 5 activity is significantly (al- 
A

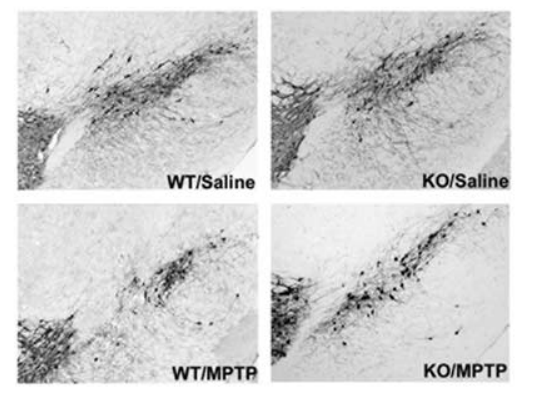

B

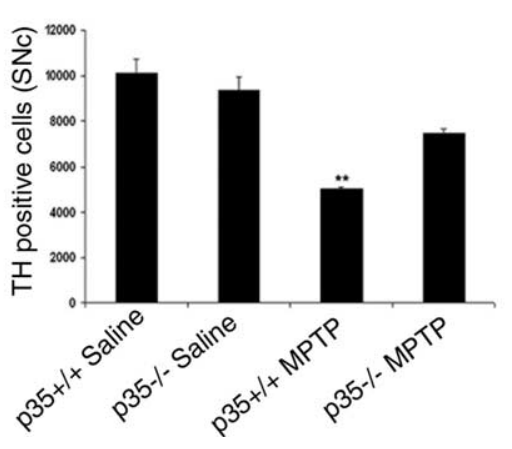

C

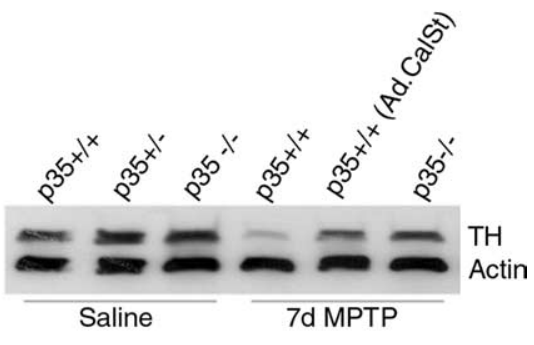

D

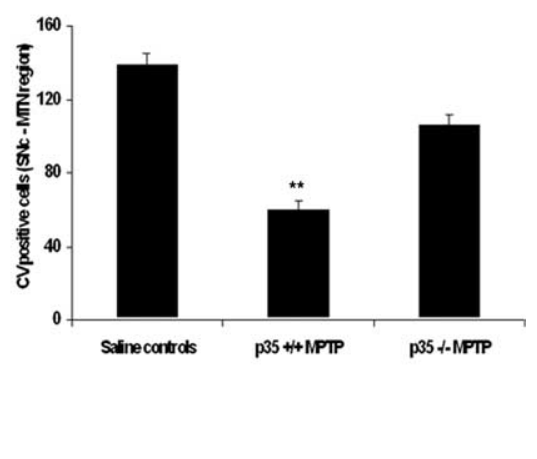

Figure 2. p35 deficiency attenuates MPTP-induced dopamine neuron loss. $\boldsymbol{A}$, Representative photomicrographs showing significant reduction in total number of TH-positive cells after MPTP treatment in wild-type mice. MPTP-induced loss of TH was attenuated in p35 mutant mice $14 \mathrm{~d}$ after MPTP treatment. B, Quantification of total TH-positive cell bodies in the SNc, after saline or chronic MPTP treatment, in p35 mutants and littermate controls reveal significant attenuation of MPTP-induced dopamine neuron loss in the substantia nigra of p35 null mice. C, Western blot analysis reveals no significant differences in TH expression ( $\sim 58 \mathrm{kDa}$ molecular weight) in nigral samples extracted from $\mathrm{p} 35$ null mice when compared with littermate control. Seven days after MPTP treatment, both p35 null mice and adenoviral calpastatin (Ad. CalSt.)-treated mice showed attenuation of MPTPinduced TH loss in the nigral region. The membrane was blotted simultaneously with $\beta$-actin (as a loading control). D, Quantification of CV-positive cells in the SNc (MTN region) of saline controls ( $\mathrm{p} 35$ wild type and p35 null; $n=7$ ) and MPTP-treated p35 wild-type $(n=5)$ and MPTP-treated 355 null $(n=6)$ mice. Asterisks represent $p<0.01$ (ANOVA; Newman-Keuls post hoc; $n=$ 6 per treatment group). K0, Knock-out mice; WT, wild-type mice. Error bars represent \pm SEM.

though not completely) reduced by calpain inhibitors, calpains likely play a critical role in the observed $c \mathrm{dk} 5$ activation.

\section{p35-deficient mice show attenuation of MPTP-induced dopamine neuron loss in vivo}

Because our data indicated that calpains modulate the cdk5/p35 complex in response to MPTP treatment, resulting in overactivation of cdk5 in the substantia nigra (Fig. 1), we were interested in examining the effect of $\mathrm{p} 35$ deficiency on the survival of dopaminergic neurons. p35-deficient mice were originally generated by Hallows et al. (2003). p35-deficient mice display some CNS developmental abnormalities (Hallows et al., 2003). No gross abnormalities were detected in the dopamine system of these mice. First, no significant difference in number of nigral neurons in p35-deficient mice was observed when compared with wild-type littermates (Fig. $2 A, B$ ). Second, there was no difference in basal locomotor activity of p35 mutants when compared with wildtype littermates (Fig. $4 B$ ). Interestingly, recent reports indicated that $\mathrm{TH}$, the rate-limiting enzyme in dopamine synthesis and a phenotypic marker of dopamine neurons, was phosphorylated by cdk5, resulting in increased total cdk5 activity (Moy and Tsai, 2004). To evaluate the potential effect of p35 deficiency on $\mathrm{TH}$ expression, we used Western blot analysis to examine the levels of $\mathrm{TH}$ in the nigral region of p35 null mice and wild-type littermates. No significant difference in basal TH expression was observed in the nigral region of p35-deficient mice when compared with wild-type animals (Fig. 2C). Consistent with this result, no difference in striatal dopamine levels was detected between wild-type and p35deficient mice (Fig. 3).

After MPTP treatment, the level of TH protein in the substantia nigra at $7 \mathrm{~d}$ (Fig. $2 C)$, as well as the number of TH-positive neurons at $14 \mathrm{~d}$ (Fig. 2A), were significantly decreased in wild-type mice after MPTP treatment. p35-deficient mice showed significant attenuation of this MPTP-induced TH loss in the nigra at $7 \mathrm{~d}$ (Fig. 2C). Similar attenuation of MPTPinduced $\mathrm{TH}$ loss was also observed in calpastatin-treated nigral samples (Fig. $2 C$ ), in agreement with our previous report (Crocker et al., 2003). When evaluated at $14 \mathrm{~d}, \mathrm{p} 35$-deficient mice showed significant attenuation of $\mathrm{TH}$-positive neuron loss when compared with wildtype mice (Fig. 2A,B). To address the issue of potential loss of $\mathrm{TH}$ (a phenotypic marker of dopamine neurons) without loss of dopaminergic cells in the nigral region, we stained nigral sections with $\mathrm{CV}$, an independent histological marker. Consistent with the observed TH-positive cell counts, there was a significant reduction in the number of cresyl violet-positive neurons in the SNc of MPTP-treated wild-type littermates (Fig. 2D). This MPTP-induced reduction in CV-positive cells was attenuated in p35 mutant mice (Fig. 2D), mirroring the observed TH-positive cell counts.

\section{Effect of p35 deficiency on the striatal output region}

Dopaminergic cells in the SNc project fibers mainly to the striatal output region. Given that we observed a protective effect on nigral dopamine cell bodies in p35-deficient mice after MPTP treatment, we next examined whether this resulted in preservation of dopaminergic fibers of the striatum. No significant difference in striatal DAT (a phenotypic dopamine neuron marker) expression was observed in untreated p35 mutant nigra when compared with control wild-type littermates (Fig. 3). Strikingly, p35-deficient mice displayed significant improvement in the expression of DAT in the striatum, when compared with p35 wildtype littermates treated with MPTP (Fig. $3 A-E$ ). Moreover, the preservation in dopaminergic fibers correlated with increased dopamine levels in MPTP-treated p35 mutants as measured by HPLC analysis of striatal extracts (Fig. $3 F$ ).

When injected intraperitoneally into mice, MPTP crosses the blood-brain barrier and is converted to its active metabolite, $\mathrm{N}$-methyl-4-phenylpyridinium ion $\left(\mathrm{MPP}^{+}\right)$, by monoamine oxidase (Heikkila et al., 1984; Javitch et al., 1985). Hence, it is important to determine whether observed protection in p35deficient mice is attributable to altered formation of the active toxin. We found no significant differences in $\mathrm{MPP}^{+}$levels in the different genotypes $90 \mathrm{~min}$ after MPTP treatment (Fig. 3G), suggesting that the absence of $\mathrm{p} 35$ does not alter the generation of the active metabolite of MPTP.

Previous evidence indicates that increase of $\delta$ FosB, an immediate early gene that is upregulated with striatal denervation and changes in postsynaptic signaling (Crocker et al., 2003; Smith et 
al., 2003). Consistent with previous results, MPTP treatment resulted in a significant induction of $\delta$ FosB expression in the striatum (Fig. 4A). This effect was likely induced as a consequence of hypersensitization of the terminals after denervation. Importantly, p35 null mice showed significantly less total $\delta$ FosB expression after MPTP treatment when compared with WT controls (Fig. 4A), in further support of the evidence that the striatal output fibers were functionally preserved in p35 null mice after MPTP treatment. It must be noted that p35 null mice appeared to start out with a slightly lower basal level of FosB; however, this difference was not statistically significant.

Finally, to further examine the functional implications of p35 deficiency, we examined behavioral responses after MPTP treatment. We previously reported that MPTP significantly impairs locomotor activity (Crocker et al., 2003; Smith et al., 2003). We therefore performed experiments to evaluate the effect of p35 deficiency on gross locomotor activity, using a computer assisted home-cage behavior apparatus. Gross locomotor activity was analyzed in the home cage for $24 \mathrm{~h}$ in the beam break system before initiation of MPTP treatment (basal) and 7 and $14 \mathrm{~d}$ after MPTP administration. We show in Figure 4 that p35deficient mice did not display any gross abnormalities in locomotor activity, as measured by the beam break system. However, after treatment with MPTP, the p35 null mice showed significant attenuation of MPTP-induced hypolocomotion at 7 and $14 \mathrm{~d}$ after MPTP treatment (Fig. 4 B). Combined with preservation of dopamine neurons and increased dopamine levels, this behavioral data indicate that, indeed, a p35-mediated response is involved in functional preservation of the nigrostriatal pathway after neurotoxic insult. Although these data collectively elucidate the importance of a calpain-cdk5-mediated pathway in dopamine loss, the mechanism downstream of cdk5 is unknown.

\section{MEF2D, a target of cdk5-mediated phosphorylation, plays a} critical role in regulation of dopamine neuron damage

We previously showed that MEF2D, a transcription factor known to be involved in transcriptional activation of survival signals, is a potential target of cdk5-mediated kinase activity in vitro (Gong et al., 2003). In certain circumstances, cdk5 phosphorylates MEF2D on the Serine 444 residue and results in inactivation of the transcription factor (Gong et al., 2003). The role of MEF2 in dopamine survival in vivo as well as its relationship to the calpain/cdk5 pathway in dopaminergic loss is unknown. Thus, we determined whether calpain activation is required for cdk5-mediated phosphorylation of MEF2D and whether inhibition of this event protects dopamine neurons after MPTP treatment. We first evaluated the phosphorylation state of MEF2D using a phosphoepitope antibody that recognized the Serine 444 site (pMEF2D) in the substantia nigra of mice after MPTP treatment. Using Western blot analysis and immunohistochemical approaches on nigral samples, we found that MPTP induced an increase in phosphorylation of MEF2D in the substantia nigra region (Fig. 5A).
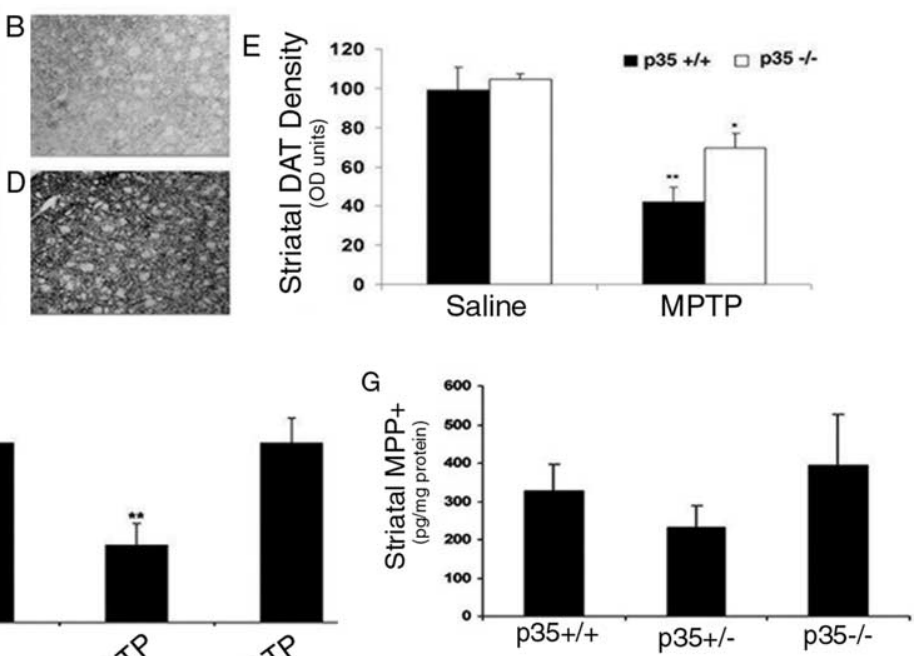

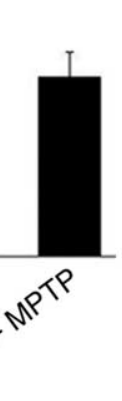$$
\text { . }
$$

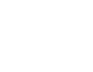

Figure 3. p35 null mice show improvement in MPTP-induced loss of striatal function. $A, C$, Representative photomicrographs show that p35 null mice display normal expression of DAT. $\boldsymbol{B}$, Significant reduction in DAT expression was observed after MPTP

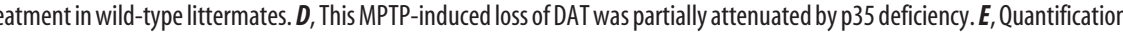
(n) treatment group). G, Striatal MPP ${ }^{+}$measurement 90 min after a single $30 \mathrm{mg} / \mathrm{kg}$ MPTP injection shows no significant differences in metabolism of MPTP in the different genotypes ( $n=4$ per treatment group). ${ }^{*} p<0.05$, ANOVA; ${ }^{* *} p<0.01$, ANOVA Newman-Keuls post hoc test. Error bars represent \pm SEM.
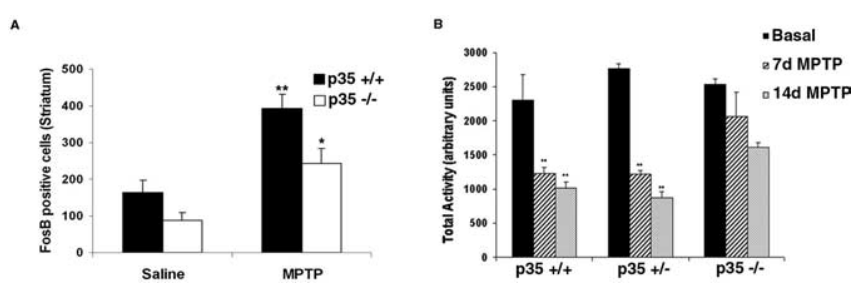

Figure 4. p35-deficient mice show significant attenuation of MPTP-induced increase in markers of denervation and behavioral deficits. $A$, Quantification of $\delta$ FosB expression in the striatal region after saline or MPTP treatment in wild-type and p35 null mice ( $n=4-5$ per treatment group). $\boldsymbol{B}$, Home cage "beam break" locomotor activity of $\mathrm{p} 35$ wild-type and mutant mice after saline or MPTP treatment show that $\mathrm{p} 35$ deficiency alone did not influence locomotor activity; p35 deficiency attenuates MPTP-induced behavioral deficits. The bar graph represents total locomotor activity before initiation of MPTP treatment (basal) and 7 and $14 \mathrm{~d}$ after MPTP treatment. Activity was monitored over a $24 \mathrm{~h}$ period ( $n=6-11$ per group). ${ }^{*} p<0.05$, ANOVA, ${ }^{* *} p<0.01$, ANOVA; Newman-Keuls post hoc test. Error bars represent \pm SEM.

Double immunofluorescence analysis with TH and pMEF2D revealed that pMEF2D is expressed in dopaminergic neurons (data not shown). We also showed that there was increased formation of a cleavage product of the MEF2D protein (Fig. $5 \mathrm{~A}$ ). Cleavage of MEF2D has been described previously and is suggested to be mediated by its phosphorylation state and caspase-dependent cleavage (Li et al., 2001; Tang et al., 2005). We next determined whether the observed MEF2D phosphorylation event was dependent on a potential calpain-mediated $\mathrm{cdk} 5 / \mathrm{p} 35$ response. We found that MPTP-induced phosphorylation of MEF2D was reduced with calpain inhibition as well as the absence of p35 (Fig. $5 A$ ). It must be noted that the dynamics and relationship between MEF2D phosphorylation and cleavage is not clear and cannot be fully determined from our present studies. However, it does ap- 
A.

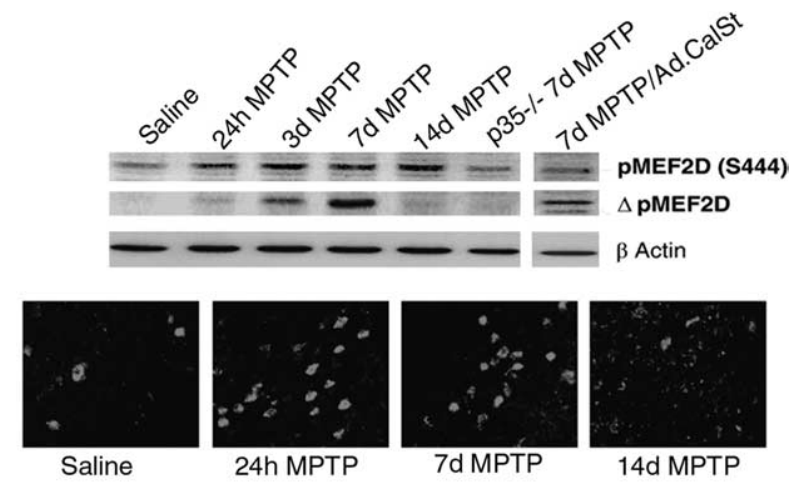

B

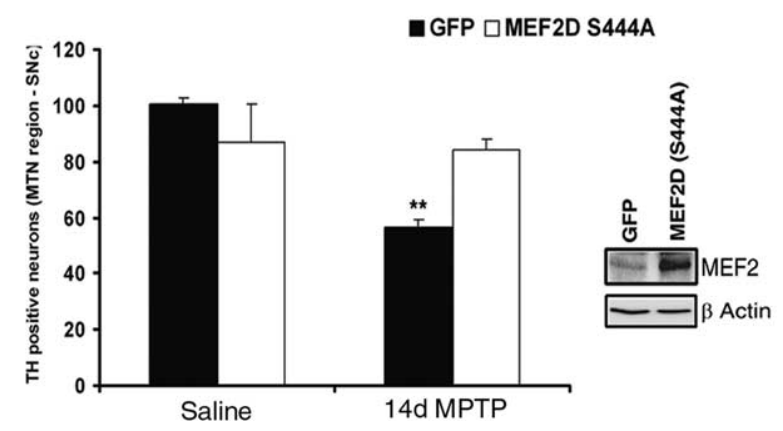

Figure 5. MEF2D is a critical downstream target of cdk5 that is induced in response to MPTP. $A$, Representative Western blot analysis showing an increase in phosphorylation of MEF $2 D$ at Serine 444 (pMEF2D) in nigral samples extracted $24 \mathrm{~h}, 3 \mathrm{~d}$, and $7 \mathrm{~d}$ after the first MPTP injection. MPTP also induced cleavage of pMEF2D, which is dependent on p35, because p35 null mice show reduced PMEF2D and reduced formation of the cleavage product; similar results were obtained with calpain inhibition (Ad. CalSt.) at $7 \mathrm{~d}$ after MPTP. Similar results were obtained in three independent experiments. Immunofluorescence analysis reveals that PMEF2D is expressed in the nigral region after MPTP treatment. $\boldsymbol{B}$, Quantification of TH-positive neurons in the SNc (MTN anatomical region) after MEF2D (S444A) mutant or GFP adenoviral protein expression with saline or MPTP treatment ( $n=6$ per group). C, Inset, Western blot analysis showing increased MEF2 protein expression in the nigral region of GFP-treated and MEF2D (S444A) adenovirus-treated mice. ${ }^{* *} p<0.01$, ANOVA; Newman-Keuls post hoc test. Error bars represent \pm SEM.

pear that both are significantly reduced (although not completely eliminated) with calpain inhibition or p35 deficiency (Fig. 5A).

To determine whether MEF2s play a potential functional role in dopamine cell damage after MPTP treatment, we expressed a mutant form of MEF2D (S444A) by an adenoviral-mediated approach [Ad. MEF2D (S444A)] and examined the effect on dopamine neuron survival. Expression of adenoviral MEF2 protein was verified by Western blot analysis (Fig. $5 B$, inset). We found that unilateral expression of MEF2D (S444A) resulted in significant dopaminergic neuroprotection when compared with the ipsilateral side of GFP-treated controls that received MPTP (Fig. $5 B$ ). Overexpression of MEF2C (MEF2CVP16), a constitutively active form of MEF2C, also showed similar effects on dopaminergic neuroprotection after MPTP treatment (data not shown). These data indicate for the first time that MEF2s can modulate dopamine neuron survival in the substantia nigra after MPTP treatment. Together, the data support a critical role for a calpainregulated $\mathrm{p} 35 / \mathrm{cdk} 5$ complex in dopaminergic neuron damage through modulation of MEF2D phosphorylation and subsequent cleavage.

\section{Discussion}

Calpains are proteases thought to be important in regulation of several physiological processes, including a potential role in neu- ronal death (Goll et al., 2003). Although interesting, much of the data available exploring a potential role for calpains in neuronal death rely significantly on the use of pharmacological agents that may impact nonspecific targets. To elucidate a more specific role for calpains in death processes in an adult in vivo system, we recently used an adenoviral-mediated approach to specifically inhibit calpains in the nigrostriatal pathway by expression of calpastatin; we found that calpastatin expression attenuated MPTPinduced dopamine cell loss and improved behavioral deficits induced by MPTP (Crocker et al., 2003). Although these data provided striking evidence supporting a role for calpains in dopamine neuron damage, the targets of calpains in the CNS have not been defined clearly. Recent evidence indicate that one potential CNS-specific target of calpains is the cdk 5 activator, p35 (Kusakawa et al., 2000; Lee et al., 2000). Whether this process plays a significant role in neurodegeneration remains a subject of considerable debate.

Our present work defines a calpain-mediated pathway that regulates dopaminergic neurodegeneration in an in vivo model of PD. We show that MPTP induces calpain-dependent modulation of the $\mathrm{p} 35 / \mathrm{cdk} 5$ complex in the substantia nigra region (Fig. 1). It is important to note that, whereas p 25 formation has been described in the in vivo models of neurodegeneration, the functional implications are not clear. Here, we provide in vivo evidence that not only is calpain required for $\mathrm{p} 25$ formation, but it is also likely central to increased cdk 5 activity after neurotoxic insult.

How might p25 formation regulate a death process? p 25 is reported to be more stable and leads to higher cdk 5 activity than p35 (Hisanaga and Saito, 2003). This is consistent with our observed increase in cdk5 activity after MPTP treatment, which is blocked by calpastatin expression. However, in this case, it is important to also acknowledge other mechanisms of cdk5 control such as with phosphorylation modifications that may impact cdk5 activity (Sharma et al., 1999; Rosales et al., 2003). It is also possible that calpain-mediated generation of p25 leads to differential recognition of cdk5 substrates. This is substantiated by evidence that the full-length $\mathrm{p} 35$ protein has a myristoylation sequence, which tethers the protein to the intracellular cell membrane (Hisanaga and Saito, 2003). Because p25 is a C-terminal fragment of p35, it has been suggested that calpain-mediated proteolysis results in loss of the N-terminal myristoylation sequence, resulting in mislocalization of cdk5 kinase activity. In support of this possibility, previous evidence suggested that cdk5 can phosphorylate nuclear targets in the context of neurotoxicity in vitro (Gong et al., 2003). This suggests that nuclear events may be central to a potential pathogenic cdk 5 function. In support of this, we show here that neurotoxicity induced by MPTP, in vivo, increases phosphorylation of the transcription factor, MEF2D on a cdk5 specific site (Fig. 5). We believe this MEF2 phosphorylation is a central event downstream of cdk5 activation, and the implications of this are described in more detail below. A third possibility is that loss of a $\mathrm{p} 35 / \mathrm{cdk} 5$ complex governed by calpain activity would lead to a loss of a "normal" cytoplasmic survival function of cdk5. Indeed, the ability of $\mathrm{p} 35 / \mathrm{cdk} 5$ complex to inhibit c-Jun N-terminal protein kinase 3, often associated with neuronal death, has been described previously (Li et al., 2002). In addition, we have shown that expression of wild-type cdk 5 exclusively in the cytoplasm is protective in a glutamate model of neuronal death in vitro (O'Hare et al., 2005). This suggests that both loss of a normal neuronal maintenance function as well as a gain of pathogenic cdk5 function may be important.

Previous work implicating the role of cdk5 in dopaminergic 
loss relied on pharmacological or DN CDK strategies, which could potentially function through non-CDK-related mechanisms of action. Therefore, it was critical to more directly evaluate the role of $\mathrm{p} 35 / \mathrm{cdk} 5$-mediated processes. To do this, we used p35-deficient mice. Our p35-deficient mice (C57BL/6 background) did not show any obvious defect in the dopaminergic system (Figs. 2, 3) or show any seizure activity ascribed to other p35-deficient mice on differing backgrounds (Chae et al., 1997). We found that $\mathrm{p} 35$-deficient mice displayed significant attenuation of MPTP-induced dopaminergic neuron loss (Fig. 2). Consistent with preservation of dopaminergic fibers and increased dopamine levels in the striatal region (Fig. 3), p35-deficient mice also showed improvement in MPTP-induced behavioral deficits and improved postsynaptic signaling (Fig. 4). These data indicate the critical nature of $\mathrm{p} 35 / \mathrm{p} 25$-mediated signals in dopaminergic loss in vivo. Importantly, because our data also indicate that MPTP-induced increase in cdk5 activity is inhibited by calpastatin as well as p35 deficiency, we contend that a calpain-mediated modulation of p35 is central to and required for dopaminergic loss. Interestingly, the data also suggest that, at least in dopaminergic neurons exposed to MPTP, the pathogenic role of cdk5 (likely mediated by p25) outweighs any potential beneficial protective signal provided by the normal $\mathrm{p} 35 / \mathrm{cdk} 5$ complex.

Although these findings suggest a model by which p35 deficiency is protective by abolishing the production of a toxic $\mathrm{p} 25 /$ cdk5 complex, there are obvious caveats with use of germlinedeficient animals. For example, the long-term absence of p35 may have unapparent secondary effects that affect dopamine neuron response to MPTP. However, the present results are consistent with our previous findings indicating that DN cdk5 expression also blocked MPTP-induced neuronal loss (Smith et al., 2003). In addition, although these data point to a role for calpainmediated modulation of cdk5/p35 in dopaminergic cell loss, it is important to note that cdk 5 can be activated by $\mathrm{p} 39$ (Humbert et al., 2000). Whether p39 plays a role in MPTP-induced loss remains to be examined. However, our observation that p35 deficiency significantly blocked induction of cdk 5 activity observed with MPTP, as well as observations indicating that p39 mice have a milder phenotype than $\mathrm{p} 35$-deficient mice, suggest a predominate role for p35 in dopaminergic cell death processes. It is also important to point out that although we have focused on a calpain-p35 pathway of death in the MPTP model, calpains can potentially target other cellular processes that may impact on dopaminergic cell death processes. Of particular relevance, we previously demonstrated that calpains mediate $\mathrm{p} 53$ activation in vitro (Sedarous et al., 2003), and others have reported resistance of p53-deficient mice to MPTP insult (Trimmer et al., 1996). Finally, we must emphasize that we have not demonstrated conclusively the activation of the calpain-p35-cdk5-MEF2 pathway in dying dopaminergic neurons. We found it difficult to identify neurons in the late stage of death with this model, a limitation of our present study. This is likely because of the sporadic/protracted time frame of death initiation in the substantia nigra. Therefore, although we hypothesize that these processes are autonomous to dopamine neurons, this is not completely clear from the present data. The data do, however, suggest that modulation of the calpain-cdk5-MEF2 pathway, at several levels, results in improved survival of dopamine neurons after MPTP treatment.

Although these data substantiate a role for calpain-regulated $\mathrm{p} 35 / \mathrm{cdk} 5$ in dopaminergic cell death processes, the downstream pathogenic effects of this process is not clear. Recent evidence indicated that MEF2D is phosphorylated by cdk5 on an inactivat- ing site after neurotoxic insult in vitro (Gong et al., 2003). In addition, others have reported that hyperphosphorylation of MEF2D is associated with apoptosis (Li et al., 2001) and could significantly regulate MEF2 transcriptional activity (Gong et al., 2003). Finally, it is suggested that phosphorylation of MEF2 is a critical primer for caspase-dependent cleavage and generation of dominant-negative interfering forms of MEF2, which may mediate death signaling events (Mao and Wiedmann, 1999; Li et al., 2001). Because previous data indicate that cdk5 can target MEF2D (Gong et al., 2003), and given the potential calpaindependent influence on cdk5-mediated responses, we were interested in determining whether MEF2D played a role in dopaminergic cell loss and whether this signal was dependent on calpain activation. We report here that MPTP induced cdk5-mediated phosphorylation of MEF2D on Serine 444 (an inactivating site), which is subsequently associated with formation of a MEF2D cleavage product. This induction in phosphorylation is dependent, at least in part, on the activation of calpains (Fig. 5). The absence of $\mathrm{p} 35$ also reduces the phosphorylation (and subsequent cleavage) of MEF2D, suggesting that a p35/cdk5-mediated signal is involved in this process. Although both phosphorylation of MEF2D and cleavage occurs in the substantia nigra after MPTP treatment, it is not clear whether cdk5-mediated phosphorylation is a direct prerequisite for MEF2D cleavage. However, given previous evidence that hyperphosphorylation of MEF2 is associated with cleavage by caspases (Nakamura et al., 1997), we suggest that cdk5-mediated MEF2 phosphorylation leads to inactivation and subsequent caspase-dependent cleavage (supplemental Fig. S1, available at www.jneurosci.org as supplemental material), resulting in suppression of MEF2 survival function. To further clarify the role of cdk5-regulated MEF2D, we show that expression of an adenoviral form of MEF2D (S444A) results in attenuation of MPTP-induced dopaminergic cell loss. Together, these data define a novel mechanism by which calpains regulate dopaminergic neuron loss through modulation of the cdk5/p35 complex in an adult in vivo model of PD.

\section{Concluding remarks}

The results presented here suggest that a calpain-p35/cdk5-MEF2 pathway plays a central role in dopaminergic death in a rodent model of PD. Whether this is true of human PD is difficult to ascertain. However, there are some tantalizing clues that the MPTP model may recapitulate similar pathways in humans. For example, increased expression of calpains and increased calpain activity has been observed in dopaminergic neurons of postmortem PD brains. Cdk5 has also been reported to be elevated in postmortem brains of PD patients. Given these similarities, we propose that targeting and specifically inhibiting the calpainp35/cdk5-MEF2 cascade may be effective in protecting dopamine neurons in PD.

\section{References}

Abercrombie M (1946) Estimation of nuclear population from microtome sections. Anat Rec 94:239-247.

Bu B, Li J, Davies P, Vincent I (2002) Deregulation of cdk5, hyperphosphorylation, and cytoskeletal pathology in the Niemann-Pick type $\mathrm{C}$ murine model. J Neurosci 22:6515-6525.

Carafoli E, Molinari M (1998) Calpain: a protease in search of a function? Biochem Biophys Res Commun 247:193-203.

Chae T, Kwon YT, Bronson R, Dikkes P, Li E, Tsai LH (1997) Mice lacking p35, a neuronal specific activator of Cdk5, display cortical lamination defects, seizures, and adult lethality. Neuron 18:29-42.

Chan SL, Mattson MP (1999) Caspase and calpain substrates: roles in synaptic plasticity and cell death. J Neurosci Res 58:167-190.

Chergui K, Svenningsson P, Greengard P (2004) Cyclin-dependent kinase 5 
regulates dopaminergic and glutamatergic transmission in the striatum. Proc Natl Acad Sci USA 101:2191-2196.

Croall DE, McGrody KS (1994) Domain structure of calpain: mapping the binding site for calpastatin. Biochemistry 33:13223-13230.

Crocker SJ, Lamba WR, Smith PD, Callaghan SM, Slack RS, Anisman H, Park DS (2001) c-Jun mediates axotomy-induced dopamine neuron death in vivo. Proc Natl Acad Sci USA 98:13385-13390.

Crocker SJ, Smith PD, Jackson-Lewis V, Lamba WR, Hayley SP, Grimm E, Callaghan SM, Slack RS, Melloni E, Przedborski S, Robertson GS, Anisman H, Merali Z, Park DS (2003) Inhibition of calpains prevents neuronal and behavioral deficits in an MPTP mouse model of Parkinson's disease. J Neurosci 23:4081-4091.

Dauer W, Przedborski S (2003) Parkinson's disease: mechanisms and models. Neuron 39:889-909.

Franklin KB, Paxinos G (2001) The mouse brain in stereotaxic coordinates. New York: Academic.

Goll DE, Thompson VF, Li H, Wei W, Cong J (2003) The calpain system. Physiol Rev 83:731-801.

Gong X, Tang X, Wiedmann M, Wang X, Peng J, Zheng D, Blair LA, Marshall J, Mao Z (2003) Cdk5-mediated inhibition of the protective effects of transcription factor MEF2 in neurotoxicity-induced apoptosis. Neuron 38:33-46.

Hallows JL, Chen K, DePinho RA, Vincent I (2003) Decreased cyclindependent kinase 5 (cdk5) activity is accompanied by redistribution of cdk5 and cytoskeletal proteins and increased cytoskeletal protein phosphorylation in p35 null mice. J Neurosci 23:10633-10644.

Hayley S, Lacosta S, Merali Z, van Rooijen N, Anisman H (2001) Central monoamine and plasma corticosterone changes induced by a bacterial endotoxin: sensitization and cross-sensitization effects. Eur J Neurosci 13:1155-1165.

Hayley S, Crocker SJ, Smith PD, Shree T, Jackson-Lewis V, Przedborski S, Mount M, Slack R, Anisman H, Park DS (2004) Regulation of dopaminergic loss by Fas in a 1-methyl-4-phenyl-1,2,3,6-tetrahydropyridine model of Parkinson's disease. J Neurosci 24:2045-2053.

Heikkila RE, Manzino L, Cabbat FS, Duvoisin RC (1984) Protection against the dopaminergic neurotoxicity of 1-methyl-4-phenyl-1,2,5,6-tetrahydropyridine by monoamine oxidase inhibitors. Nature 311:467-469.

Hisanaga S, Saito T (2003) The regulation of cyclin-dependent kinase 5 activity through the metabolism of p 35 or p 39 Cdk 5 activator. NeuroSignals $12: 221-229$

Humbert S, Dhavan R, Tsai L (2000) p39 activates cdk5 in neurons, and is associated with the actin cytoskeleton. J Cell Sci 113:975-983.

Javitch JA, D’Amato RJ, Strittmatter SM, Snyder SH (1985) Parkinsonisminducing neurotoxin, $N$-methyl-4-phenyl-1,2,3,6 -tetrahydropyridine: uptake of the metabolite $\mathrm{N}$-methyl-4-phenylpyridine by dopamine neurons explains selective toxicity. Proc Natl Acad Sci USA 82:2173-2177.

Julien JP, Mushynski WE (1998) Neurofilaments in health and disease. Prog Nucleic Acid Res Mol Biol 61:1-23.

Ko J, Humbert S, Bronson RT, Takahashi S, Kulkarni AB, Li E, Tsai LH (2001) p35 and p39 are essential for cyclin-dependent kinase 5 function during neurodevelopment. J Neurosci 21:6758-6771.

Kusakawa G, Saito T, Onuki R, Ishiguro K, Kishimoto T, Hisanaga S (2000) Calpain-dependent proteolytic cleavage of the p35 cyclin-dependent kinase 5 activator to p25. J Biol Chem 275:17166-17172.

Lee MS, Kwon YT, Li M, Peng J, Friedlander RM, Tsai LH (2000) Neurotoxicity induces cleavage of $\mathrm{p} 35$ to $\mathrm{p} 25$ by calpain. Nature 405:360-364.

Li BS, Zhang L, Takahashi S, Ma W, Jaffe H, Kulkarni AB, Pant HC (2002) Cyclin-dependent kinase 5 prevents neuronal apoptosis by negative regulation of c-Jun N-terminal kinase 3. EMBO J 21:324-333.

Li M, Linseman DA, Allen MP, Meintzer MK, Wang X, Laessig T, Wierman ME, Heidenreich KA (2001) Myocyte enhancer factor 2A and 2D undergo phosphorylation and caspase-mediated degradation during apoptosis of rat cerebellar granule neurons. J Neurosci 21:6544-6552.

Mao Z, Wiedmann M (1999) Calcineurin enhances MEF2 DNA binding activity in calcium-dependent survival of cerebellar granule neurons. J Biol Chem 274:31102-31107.

Melloni E, Salamino F, Sparatore B (1992) The calpain-calpastatin system in mammalian cells: properties and possible functions. Biochimie 74:217-223.

Mouatt-Prigent A, Karlsson JO, Agid Y, Hirsch EC (1996) Increased
M-calpain expression in the mesencephalon of patients with Parkinson's disease but not in other neurodegenerative disorders involving the mesencephalon: a role in nerve cell death? Neuroscience 73:979-987.

Moy LY, Tsai LH (2004) Cyclin-dependent kinase 5 phosphorylates serine 31 of tyrosine hydroxylase and regulates its stability. J Biol Chem 279:54487-54493.

Nakamura S, Kawamoto Y, Nakano S, Akiguchi I, Kimura J (1997) p35nck5a and cyclin-dependent kinase 5 colocalize in Lewy bodies of brains with Parkinson's disease. Acta Neuropathol (Berl) 94:153-157.

Nguyen MD, Lariviere RC, Julien JP (2001) Deregulation of Cdk5 in a mouse model of ALS: toxicity alleviated by perikaryal neurofilament inclusions. Neuron 30:135-147.

Nishi A, Bibb JA, Matsuyama S, Hamada M, Higashi H, Nairn AC, Greengard P (2002) Regulation of DARPP-32 dephosphorylation at PKA- and Cdk5-sites by NMDA and AMPA receptors: distinct roles of calcineurin and protein phosphatase-2A. J Neurochem 81:832-841.

O'Hare MJ, Kushwaha N, Zhang Y, Aleyasin H, Callaghan SM, Slack RS, Albert PR, Vincent I, Park DS (2005) Differential roles of nuclear and cytoplasmic cyclin-dependent kinase 5 in apoptotic and excitotoxic neuronal death. J Neurosci 25:8954-8966.

Ohshima T, Ward JM, Huh CG, Longenecker G, Veeranna, Pant HC, Brady RO, Martin LJ, Kulkarni AB (1996) Targeted disruption of the cyclindependent kinase 5 gene results in abnormal corticogenesis, neuronal pathology and perinatal death. Proc Natl Acad Sci USA 93:11173-11178.

Patrick GN, Zukerberg L, Nikolic M, de la Monte S, Dikkes P, Tsai LH (1999) Conversion of p 35 to $\mathrm{p} 25$ deregulates Cdk 5 activity and promotes neurodegeneration. Nature 402:615-622.

Pines J (1993) Cyclins and their associated cyclin-dependent kinases in the human cell cycle. Biochem Soc Trans 21:921-925.

Ray SK, Banik NL (2003) Calpain and its involvement in the pathophysiology of CNS injuries and diseases: therapeutic potential of calpain inhibitors for prevention of neurodegeneration. Curr Drug Targets CNS Neurol Disord 2:173-189.

Rosales JL, Nodwell MJ, Johnston RN, Lee KY (2000) Cdk5/p25(nck5a) interaction with synaptic proteins in bovine brain. J Cell Biochem 78:151-159.

Rosales J, Han B, Lee KY (2003) Cdk7 functions as a cdk5 activating kinase in brain. Cell Physiol Biochem 13:285-296.

Sedarous M, Keramaris E, O’Hare M, Melloni E, Slack RS, Elce JS, Greer PA, Park DS (2003) Calpains mediate p53 activation and neuronal death evoked by DNA damage. J Biol Chem 278:26031-26038.

Sharma P, Sharma M, Amin ND, Albers RW, Pant HC (1999) Regulation of cyclin-dependent kinase 5 catalytic activity by phosphorylation. Proc Natl Acad Sci USA 96:11156-11160.

Shelton SB, Johnson GV (2004) Cyclin-dependent kinase-5 in neurodegeneration. J Neurochem 88:1313-1326.

Smith PD, Crocker SJ, Jackson-Lewis V, Jordan-Sciutto KL, Hayley S, Mount MP, O'Hare MJ, Callaghan S, Slack RS, Przedborski S, Anisman H, Park DS (2003) Cyclin-dependent kinase 5 is a mediator of dopaminergic neuron loss in a mouse model of Parkinson's disease. Proc Natl Acad Sci USA 100:13650-13655.

Smith PD, O'Hare MJ, Park DS (2004) CDKs: taking on a role as mediators of dopaminergic loss in Parkinson's disease. Trends Mol Med 10:445-451.

Tang X, Wang X, Gong X, Tong M, Park D, Xia Z, Mao Z (2005) Cyclindependent kinase 5 mediates neurotoxin-induced degradation of the transcription factor myocyte enhancer factor 2. J Neurosci 25:4823-4834.

Tatton NA, Kish SJ (1997) In situ detection of apoptotic nuclei in the substantia nigra compacta of 1-methyl-4-phenyl-1,2,3,6-tetrahydropyridine-treated mice using terminal deoxynucleotidyl transferase labelling and acridine orange staining. Neuroscience 77:1037-1048.

Trimmer PA, Smith TS, Jung AB, Bennett Jr JP (1996) Dopamine neurons from transgenic mice with a knockout of the $\mathrm{p} 53$ gene resist MPTP neurotoxicity. Neurodegeneration 5:233-239.

Tsai LH, Takahashi T, Caviness Jr VS, Harlow E (1993) Activity and expression pattern of cyclin-dependent kinase 5 in the embryonic mouse nervous system. Development 119:1029-1040.

Tsai LH, Delalle I, Caviness Jr VS, Chae T, Harlow E (1994) p35 is a neuralspecific regulatory subunit of cyclin-dependent kinase 5. Nature 371:419-423. 